Cahiers $d u$ MONDE RUSSE

\section{Cahiers du monde russe}

Russie - Empire russe - Union soviétique et États indépendants

$50 / 2-3 \mid 2009$

L'Europe orientale, 1650-1730. Crises, conflits et renouveau

\title{
Construire le socialisme par les chiffresMartine MESPOULET
}

, Paris : INED, 2008, 240 p., (Coll. Classiques de l'économie)

Morgane Labbé

\section{(Q) OpenEdition}

\section{Journals}

Édition électronique

URL : https://journals.openedition.org/monderusse/9791

DOI : 10.4000/monderusse. 9791

ISSN : $1777-5388$

\section{Éditeur}

Éditions de l'EHESS

\section{Édition imprimée}

Date de publication : 15 septembre 2009

ISBN : 978-2-7132-2260-3

ISSN : $1252-6576$

Référence électronique

Morgane Labbé, "Construire le socialisme par les chiffresMartine MESPOULET », Cahiers du monde russe [En ligne], 50/2-3 | 2009, mis en ligne le 14 janvier 2013, consulté le 03 septembre 2022. URL http://journals.openedition.org/monderusse/9791; DOI : https://doi.org/10.4000/monderusse.9791

Ce document a été généré automatiquement le 3 septembre 2022

Tous droits réservés 


\title{
Construire le socialisme par les chiffresMartine MESPOULET
}

\author{
, Paris : INED, 2008, 240 p., (Coll. Classiques de l'économie) \\ Morgane Labbé
}

\section{Martine MESPOULET, Construire le socialisme par les chiffres, Enquêtes et recensements en URSS de 1917 à 1991, Paris : INED, 2008, 240 p., (Coll. Classiques de l'économie)}

1 Issu de recherches rigoureuses dans les fonds documentaires russes et d'une réflexion qui s'est enrichie au contact de la sociologie des sciences, ce troisième ouvrage de Martine Mespoulet s'inscrit dans le prolongement de ses deux publications sur l'histoire de la statistique russe et soviétique ${ }^{63}$. La première était centrée sur le dynamisme de la tradition statistique russe formée à la fin du xIX siècle dans le cadre des zemstva; la seconde, cosignée avec Alain Blum, interrogeait la continuité de cette tradition après la révolution de 1917 jusqu'à son déclin dans les années 1930. L'auteur traite ici de la période de l'après-guerre jusqu'à la chute du régime soviétique, tout en conservant la perspective longue du xIX ${ }^{e}$ siècle. Celle-ci lui permet de questionner la durable influence de cette tradition fondatrice qu'elle relie à la problématique nouvelle de l'émergence d'une statistique proprement socialiste. Déjà présente en filigrane dans les autres publications de Mespoulet, se pose la question d'une statistique socialiste, genre spécifique associé à l'état socialiste, autrement dit à un mode centralisé, dirigiste et planifié d'administration de l'économie et de la société. Cette question est d'autant plus pertinente qu'elle fait écho à la typologie proposée par Alain Desrosières, qui met en relation formes de statistique et figures de l'État ${ }^{64}$. Or le planisme, que ce dernier associe à l'« État ingénieur ", ne le conduisait pas à envisager une figure originale de l'État socialiste. La sociologue s'inscrit donc dans son sillage, filiation revendiquée, pour rechercher les traits spécifiques de la statistique socialiste. À première vue, le titre de l'ouvrage apporte une réponse: les chiffres servent à "construire le socialisme». L'expression évoque immédiatement la place centrale et omnisciente du chiffre comme 
symbole de la puissance politique et de la réussite du socialisme, comme langage de représentation d'un monde nouveau et outil de légitimation politique et de conduite dirigiste de l'économie. Si Martine Mespoulet se risque à employer le terme «construire» - si fréquent pour qualifier la prépondérance de la statistique dans les États modernes -, elle lui donne aussi un sens précis : à partir des années 1930, il s'agit bien pour les dirigeants de faire entrer dans la grille de lecture marxiste, par le biais des classifications statistiques, la représentation d'une société diversifiée. Cette volonté dépasse la demande de légitimation des catégories officielles, elle s'inscrit comme nulle part ailleurs dans une recherche de l'efficacité du chiffre à faire advenir le réel, renversant ainsi le rapport de la statistique à la "réalité sociale", pour lui donner la fonction de représentation d'une société marxiste idéale posée comme premier niveau. On connaît l'exemple caricatural de Stalin fixant par avance le chiffre de la population que le recensement de 1937 devait établir; Mespoulet montre que c'est en définitive toute la statistique sociale qui s'est trouvée assujettie à cette mission.

2 Le livre semble induire que cette fonction de la statistique, certes à un degré exacerbé dans les années 1930, est liée à l'État soviétique comme modèle politique. L'hypothèse demanderait à être validée par d'autres expériences historiques, comme celles des démocraties populaires d'Europe de l'Est. Car ces traits distinctifs ne s'imposent pas dès la mise en place du nouveau régime. Dans les années qui suivent la révolution, dans le cadre de la nouvelle Direction centrale de la statistique de l'État bolchevik, c'est une autre statistique qui se développe. Une statistique particulièrement ambitieuse, au point que l'auteur peut parler d'effervescence statistique pour caractériser cette période sans précédent: multiplication des enquêtes, innovations méthodologiques, vaste recensement de la population, élaboration de nomenclatures professionnelles détaillées. La continuité avec la statistique des zemstva, qu'elle avait déjà abordée, est ici de nouveau mise en lumière pour rendre compte de cette période exceptionnelle de l'histoire de la statistique russe, voire européenne. Elle est approfondie ici par le choix que fait l'auteur d'interroger la marque du modèle par le biais des outils statistiques (questionnaires, techniques de sondage, etc.) - définis comme des « objets sociaux »pour en suivre la trajectoire sur les deux périodes encadrant la révolution. Cette approche s'avère plus fructueuse pour saisir les phénomènes de continuité que celle de son précédent ouvrage en collaboration avec Blum, qui partait des seules trajectoires des statisticiens. Elle montre que la statistique des zemstva non seulement précède et prépare la période postrévolutionnaire, mais qu'elle offre aussi les conditions techniques et cognitives de cet âge d'or, quand convergent, dans des circonstances politiques nouvelles, les expériences acquises dans les décennies antérieures et les horizons d'attente de statisticiens issus des courants de la réforme sociale. Ces expériences multiples, de négociations et de travail avec des autorités administratives, de conciliation d'objectifs divers donnés à la statistique, de coordination et d'unification de pratiques locales, et enfin d'enquêtes auprès des paysans et des ouvriers, constituèrent un savoir-faire précieux légué aux institutions statistiques soviétiques.

3 L'évolution de cette tradition peut paraître paradoxale puisqu'elle s'épanouit sous un régime qui la soumet aussi à des exigences politiques qui conduiront finalement à son déclin dans les années 1930. À partir de ce moment-là se met en place à proprement parler la statistique socialiste. Un autre atout de la démarche adoptée par l'auteur, qui étudie toujours cette évolution à partir des outils statistiques, est de ne pas réduire le nouveau rapport qui s'institue désormais entre statistique et politique en le traitant 
uniquement en termes d'assujettissement, de répression ou de manipulation. La disparition de la tradition des zemstva conduit Mespoulet, par contraste, à disqualifier la statistique qui lui succède comme non scientifique, dépourvue de finalités de connaissance et d'analyse du monde social. Elle n'en décrit pas moins des procédés et des formalismes qui attestent autant de sa transformation que de son existence. Le caractère spécifique de la statistique socialiste est, par-delà la tendance générale à la réduction des enquêtes et études sociales, la production de chiffres destinés à l'évaluation et à la prévision, et avant tout au plan. La transformation de la statistique en comptabilité, terme qui lui est désormais préféré, constitue l'hypothèse forte du livre et invite évidemment à s'interroger. L'un des points qu'on aimerait voir clarifié est la notion même de comptabilité, utilisée à la fois par l'auteur et les acteurs qu'elle étudie, dans une démarche tout autant de définition d'un type de statistique que de distinction avec un type antérieur - et qui n'est donc pas sans implicite normatif. Le terme désigne par ailleurs, dans d'autres pays, des procédés statistiques élaborés, comme la comptabilité nationale en France indexée à une politique économique. L'ouvrage ne parvient pas ici à bien spécifier l'activité statistique dont il s'agit. La finalité politique et instrumentale de cette statistique socialiste, pourtant présentée comme un trait distinctif, pourrait aussi être interrogée dans un cadre comparatif. En effet, cette fonction a été pendant plus d'un siècle remplie par le recensement américain ${ }^{65}$. Dans d'autres pays, comme la France, la statistique publique qui s'est développée dans les administrations s'apparente à cette statistique indexée sur des usages administratifs et décisionnels. Enfin, comme l'a montré Desrosières, les économistes français - théoriciens, experts ou décideurs - ont souvent élaboré une définition de la statistique tournée vers la production de chiffres pour leurs modèles et dépourvue de propriétés analytiques. Autrement dit, la tradition pointée par Mespoulet et à laquelle mirent fin les autorités soviétiques, est une tradition bien attestée qui se consolide dans toute l'Europe au XIX ${ }^{\mathrm{e}}$ siècle avec ses revendications comme science et discipline autonomes, mais il convient de rappeler qu'elle fut, dans tous les États, prise dans des tensions avec d'autres courants, comme l'économie et la sociologie naissante, qui lui refusaient ce statut et la concevaient comme une simple méthode.

Il faut néanmoins rapporter ces considérations conclusives de l'auteur au rôle qu'elle leur donne dans l'historiographie de la statistique russe où il importe de marquer une césure dans la perspective longue laissant jouer continuités et ruptures à l'écart des grandes chronologies politiques et événementielles. D'ailleurs, l'étude de deux domaines statistiques particuliers, les enquêtes par sondage et celles sur les budgets familiaux, conduit Mespoulet à mettre en relief des traits plus mélangés et contrastés qui tempèrent cette première définition tranchée de la statistique soviétique.

5 Dans ses précédentes publications, la sociologue avait déjà exposé l'essor remarquable, et longtemps méconnu, des travaux théoriques et pratiques des statisticiens russes sur la méthode des sondages, l'inscrivant toujours dans la même perspective longue. Elle interroge ici l'originalité de ce développement dans le cadre de sa problématique politique resserrée sur les outils et décrit comment les pratiques innovantes procèdent des principes socialistes. Les enquêtes sur les budgets des familles et les budgets-temps constituent une part importante et remarquable du livre, qu'elle traite selon la même approche. Martine Mespoulet montre avec précision comment, à rebours de la conception d'une statistique repliée sur la collecte de quelques données de cadrage, ces enquêtes - qui répondaient à une demande d'indices, sur le niveau de vie notamment, 
demande indéniablement politique - fournirent dans l'après-guerre des sources que la sociologie réinstituée comme science exploita avec profit.

Enfin, l'un des traits les plus étonnants mis en lumière par l'auteur, et qui donne toute la mesure de son talent à travailler ces matériaux austères, reste cette figure insolite du statisticien-enquêteur. L'importance donnée dès l'époque des zemstva à la participation du statisticien à l'enquête peut s'expliquer par divers motifs, comme le besoin d'observations complémentaires pour combler les problèmes d'incompréhension, ou la nécessité de vérifier des informations dans un contexte marqué par des suspicions réciproques. Mais il est frappant de constater que cette valeur persiste non seulement durant l'entre-deux-guerres mais également pendant l'après-guerre, quand le statisticien participe à la vie du kolkhoze. Cette particularité soulève de nombreuses questions que l'auteur ne manque pas non plus d'aborder. Car, par-delà la question de la fiabilité des informations, on peut y voir une proximité qui s'est maintenue avec la tradition monographique de la statistique européenne. On constate en dernier ressort que la spécificité de la statistique russe, qu'elle soit politique ou nationale, ne se laisse pas étudier en dehors des échanges et des circulations avec les autres traditions européennes. Mespoulet, en retraçant cette longue histoire, offre avec ce dernier livre une ouverture stimulante et riche pour une histoire européenne de la statistique jusqu'à l'Oural...

\section{NOTES}

63. Martine Mespoulet, Statistique et révolution en Russie: Un compromis impossible (1880-1930), Rennes: Presses universitaires de Rennes, 2001 ; Martine Mespoulet, Alain Blum, L'anarchie bureaucratique : Statistique et pouvoir sous Staline, P. : La Découverte, 2003.

64. Alain Desrosières, «Historiciser l'action publique: l'État, le marché et les statistiques", Pour une sociologie historique de la quantification, P.: Presses de l'École des mines, 2008, p. 39-56.

65. Voir sur ce sujet le livre de Paul Schor, Compter et classer: Histoire des recensements américains, P. : Éditions de l'EHESS, 2009. 